\title{
Future Perspectives on New Approaches in Pathogen Detection
}

\author{
Peng $\mathrm{Li}^{1}$, Bow $\mathrm{Ho}^{2}$ and Jeak Ling Ding ${ }^{3, \dagger}$ \\ ${ }^{1}$ Centre for Biomedical and Life Sciences, Singapore Polytechnic, 500 Dover Road, 139651, Singapore \\ ${ }^{2}$ Department of Microbiology, Yong Loo Lin School of Medicine, National University of Singapore 117545, Singapore \\ ${ }^{3}$ Department of Biological Sciences, National University of Singapore 117543, Singapore
}

Microbial pathogens are responsible for most of the rapidly-spreading deadly infectious diseases against humans. Thus, there is an urgent need for efficient and rapid detection methods for infectious microorganisms. The detection methods should not only be targeted and specific, but they have to be encompassing of potential changes of the pathogen as it evolves and mutates quickly during an epidemic or pandemic. The existing diagnostics such as the antibody-based ELISA immunoassay and PCR methods are too selective and narrowly focused; they are insufficient to capture newly evolved mutant strains of the pathogen. Here, we introduce a fresh perspective on some new technologies, including aptamers and next generation sequencing for pathogen detection. These technologies are not in their infancy; they are reasonably mature and ready, and they hold great promise for unparalleled applications in pathogen detection.

Key Words: Pathogen detection, Pathogen mutation, Antibiotic resistance, Aptamers, Next generation sequencing

\section{INTRODUCTION}

Emerging infectious diseases have plagued the human society globally. The world has become a playground for pathogens to develop drug resistance. The 2014 Ebola virus outbreak caused a new epidemic in West Africa (Abbaspour et al., 2015; de La Vega et al., 2015). The deadly SARS and MERS spread rapidly worldwide, threatening a new flu pandemic (Oxford et al., 2003; Wortmann, 2015). Besides these flu viruses, the well-known superbug, methicillinresistant Staphylococcus aureus (MRSA), is a major menace, challenging public health to the extent that it is estimated to be the champion killer by 2050 , overtaking cancer-related

\footnotetext{
*Received: December 3, 2015 / Accepted: December 20, 2015

${ }^{\dagger}$ Corresponding author: Jeak Ling Ding. Department of Biological Sciences, Faculty of Science, National University of Singapore, 14 Science Drive 4, 117543, Singapore.

Tel: +65-65162776, Fax: +65-67792486

e-mail: dbsdjl@nus.edu.

(C) The Korean Society for Biomedical Laboratory Sciences. All rights reserved.
}

deaths. So grave is this concern that government bodies are being set up to deal with the growing threat (http:// www.bbc.com/news/health-30416844).

During host-pathogen interactions, parasites, fungi, bacteria, and viruses activate the hosts' Toll-like receptor (TLR) mediated signalling pathways (Akira et al., 2001; Jiang et al., 2007; Li et al., 2011). The host immune system responds to the invading signals to mount antimicrobial attacks on the microbes. The host summons its production of cytokines which induce inflammatory responses during the early phase of infection. Sometimes the host immune cells enter an immune-overdrive, becoming chronically responsive to the pathogen. This may be due to the host's inability to restore homeostasis, as multiple microbial molecules simultaneously trigger several TLR signalling pathways, leading to cytokine antagonism or cytokine synergy (Schutze et al., 2011; Liu et al., 2015), the latter of which leads to cytokine storm. Often such countermeasures are either too weak or overly strong. Either way, the host frequently suffers a losing battle. The host may stand a chance of recovery if the bugs are 
susceptible to antibiotics. However, the widespread over-dose of antibiotics has resulted in a rapid rise in new mutated pathogenic strains with rampant resistance to currently available antibiotics. Thus, new pathogenic strains are resistant to currently available antibiotics. This necessitates urgent development of more powerful antibiotics.

With the abovementioned challenges, rapid identification/ detection of mutated proteins or mutated genes, which are responsible for the antibiotic-resistance has become very important. It is hoped that the prevention and treatment strategies may be modified according to the identified target. However, conventional methods of microbial detection lack robustness in the identification of mutated strains: the mutated strains could be more fastidious to in vitro culture on agar plates/broth media, therefore they miss being detected by general microbial culture methods. For the classical PCR method of detection, the mutated DNA will require new primer designs to accurately detect the mutant sequence, but only if the mutant sequence is known. Furthermore, new mutant biomarker proteins would require different cognate antibodies to be raised for assays like ELISA or other immunoassays to detect the new mutant bugs accurately. However, without knowing the change(s) in the gene sequence and proteins expressed, results from classical methods (e.g. PCR, ELISA) will not be reliable. Thus, there is an urgent need for tools that can rapidly detect and characterize mutations for accurate identification of new bugs, which will enable efficient management of infections.

Traditionally, antibiotic susceptibility determination relied on phenotypic methods, which are relatively inexpensive but may be time-consuming due to slow microbial growth under the test conditions. There is an obvious need for more robust and effective identification and characterization of possible infectious agents causing clinical syndromes, especially in patients with unknown pathogens. Thus, the sequencing of the whole genome of the pathogen is necessary. This will enable early diagnosis, prevention and the treatment of the acute infectious disease, which is crucial to stamp out a potential deadly pandemic. However, the early diagnoses of pathogenic infections in clinics have been largely ineffective and very slow. The test methods have been limited to expensive immunoassays like antibody-based enzyme-linked immunosorbent assay (ELISA) or the molecular biological assays like real-time polymerase chain reaction (qRT-PCR). These assays do not achieve cost-effective diagnostics and real-time detection required, and thus, fail to provide a timely intervention to control the infection. Thus, there is an immediate biomedical need for developing cutting-edge diagnostic tools against the new evolving pathogens. The development of these diagnostics for pathogenic infections also provides for commercial opportunities and exploitation. Currently, the new generation of molecular biotechnologies such as aptamer technology, next generation sequencing, microarray analysis, and surface plasmon resonance analysis appear to offer great promises to provide essential, high throughput and rapid pathogen-detection information to help resolve infection outbreaks.

\section{Can aptamers provide more superior pathogen diagnostics than antibodies?}

A rising generation of diagnostics and therapeutic tools based on aptamers, has been developed successfully against various pathogens (Mosing and Bowser, 2007; Ding et al., 2009; Song et al., 2012; Lee et al., 2013). Aptamers are single or double-stranded oligonucleotide molecules that bind their targets with high affinity and specificity. In principle, aptamers are one of only a few classes of molecules, which form various scaffolds and bind to the targets. The initial pool of aptamer biosensors library contains $10^{17}$ structurally different oligonucleotides. $10^{17}$ aptamers in the pool library can form hundreds of thousands of unique secondary and tertiary structures, thus a highly specific aptamer can be raised against the target of interest (like bacterial lipopolysaccharide (LPS) or viral spike protein, by undergoing the molecular evolution of selective enrichment of ligands (SELEX) (Yuce et al., 2015). During the preparation of the aptamers, the selection process for a particular target-specific aptamer is achieved in multiple rounds of SELEX and amplification, and each round of selection consists of three stages: (i) incubation of the random library pool $\left(10^{17}\right.$ aptamers $)$ with pathogen target; (ii) separation of bound and unbound aptamers by immobilization, centrifugation or microfluidics (unbound aptamers are removed by washing with a buffer 


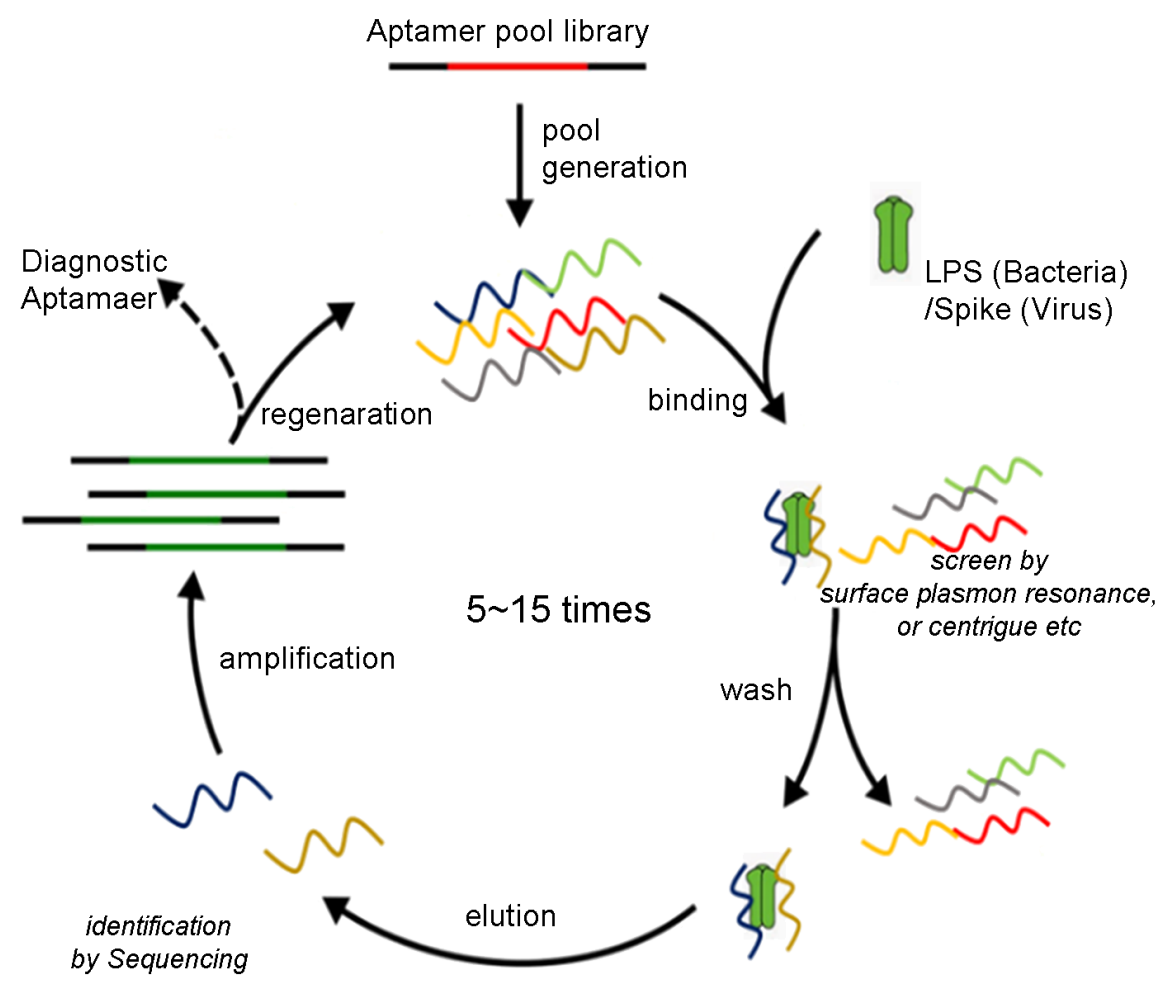

Fig. 1. The experimental approach for aptamer development. The generated pool of DNA library is exposed to the target molecule (LPS/spike protein) for binding. The washing step is used to filter out binding aptamers from non-binding aptamers. The isolated aptamers are then eluted, amplified and the process is repeated for $5 \sim 15$ rounds. After the final round of selection, aptamers are identified via functional assays and are sequenced for verification (figure is adapted and modified from (Schutze et al., 2011), with copyright permission).

solution); and (iii) elution of the bound aptamers, which are further amplified by PCR for the next round of selection. After five to fifteen rounds of SELEX, a highly specific aptamer with high affinity for the pathogen target will be enriched and concentrated. Usually the affinity of the selected aptamer to the target is increased by several orders of magnitude during each cycle of the selection process. The enriched aptamer will be sequenced. The sequenced aptamer will be synthesized and their ability to detect pathogens will be further evaluated and verified. Fig. 1. summarizes the SELEX process for the production of aptamers.

Due to the very high specificity and affinity of the selected aptamers to a target molecule, aptamers have become important diagnostic and therapeutic reagents for infectious diseases (Nimjee et al., 2005). In the published literatures, aptamers have been reported to be successfully selected, and effectively used for diagnostics and therapeutic applications
(Bruno, 2015), such as for Staphylococcus aureus diagnostics (Abbaspour et al., 2015) and acute myelogenous leukaemia cell inhibition (Kotula et al., 2014). Using a similar strategy, the aptamer biosensor for hepatitis $\mathrm{C}$ virus (HCV) has been recently developed. The aptamer biosensor has been reported to detect the HCV and other virus replication and infection in vitro and in vivo (Cho et al., 2005). The therapeutic competence of the aptamer biosensors and their exceptional targeting performance against the hepatitis virus has raised extensive interest in this class of biosensors. These studies indicate the feasibility of developing specific aptamers against pathogens and the potential of aptamers as diagnostic and therapeutic tools against infectious diseases. Our previous work attempted to develop aptamers that are ideally suited for detection of Gram-negative bacterial infection, in particular, targeting the lipopolysaccharide, LPS (Ding et al., 2009). Since the size of aptamers is 10 times smaller than large 
Table 1. List of aptamers which target microbial pathogens

\begin{tabular}{llcll}
\hline \hline Pathogens (species) & \multicolumn{1}{c}{ Microbe } & $\begin{array}{c}\text { Aptamer type } \\
\text { (DNA/RNA) }\end{array}$ & \multicolumn{1}{c}{ Application } & Reference \\
\hline Bacillus cereus & $\begin{array}{l}\text { Gram-positive } \\
\text { bacteria } \\
\text { Virus }\end{array}$ & DNA & Food pathogen Trapping & (Fischer et al., 2015) \\
Influenza & DNA & $\begin{array}{l}\text { Aptamer based microfluidic } \\
\text { diagnosis }\end{array}$ & (Lai et al., 2014) \\
E. coli O157:H7 & $\begin{array}{l}\text { Gram-negative } \\
\text { bacteria }\end{array}$ & RNA & Aptamer- immobilized ELISA & (Lee et al., 2012) \\
Mycoplasma bovis & $\begin{array}{l}\text { Mycoplasma } \\
\text { Virus }\end{array}$ & DNA & Enzyme linked aptamer detection & (Fu et al., 2014) \\
HPV-16 E7 & RNA & $\begin{array}{l}\text { HPV-infected cervical cancer } \\
\text { therapy or diagnosis }\end{array}$ & (Toscano-Garibay et al., \\
Salmonella enterica & $\begin{array}{l}\text { Gram-negative } \\
\text { bacteria } \\
\text { Gram-positive }\end{array}$ & DNA & Aptamer coupled detection sensor & (Yoo et al., 2015) \\
Staphylococcus aureus & DNA & Fluorescent labelling & (Shangguan et al., 2015) \\
\hline
\end{tabular}

Table 2. Comparison of the advantages of aptamers over antibodies

\begin{tabular}{lll}
\hline \hline & \multicolumn{1}{c}{ Antibody } & \multicolumn{1}{c}{ Aptamer } \\
\hline Chemically synthesis & Not available & Yes \\
Modification & Difficult & Easy \\
Thermostable & Denature at high temperature & Stable even at $95^{\circ} \mathrm{C}$ \\
Animal work & Dependent & Independent \\
Immunogenicity & Yes & No \\
Cost of production & Costly & Economical \\
\hline
\end{tabular}

antibodies, aptamers can be conjugated to the sensor surface at very high densities. In sample diagnostics, surface plasmon resonance only requires small sample volume, usually 20 $150 \mu \mathrm{L}$ of blood or water ( $\mathrm{Li}$ et al., 2004). Table 1 lists some DNA/RNA aptamers developed recently.

Besides individual specific aptamers, each targeting a specific domain/epitope of a pathogen, the library of aptamers for a certain pathogenic species may even be immobilized on a chip to capture multiple epitopes /antigens mimicking those on the pathogen (Zhou et al., 2015). As compared to single antigen /epitope detection, this "array of aptamerson-chip" (Lin et al., 2015) would be able to provide multiprong detection of a particular pathogen with high specificity and affinity, to raise and broaden the level of accuracy and sensitivity of detection of the pathogen. It will have consider- able applications and potential for commercial exploitation. First of all, the aptamers would be used in rapid diagnostics application at the point-of-care in pathogens testing (Lee et al., 2015). Aptamer point-of-care testing allows suspected patient diagnoses at the customs, the ambulance, the clinic, and potentially even at home. The results would be available immediately allowing the clinicians to treat the patients in a timely manner.

Moreover in the diagnostic practice, the aptamer biosensors have an edge over the competitors like antibodies (Acquah et al., 2015). There are numerous advantages with the aptamers over that of antibodies (Table 2), such as thermostability, lack of immunogenicity, and low-cost of production. Aptamers in the future are likely to fully replace antibodies in pathogens detection, especially when effective 
and specific binding to pathogenic target is identified.

\section{Next Generation Sequencing, a robust tool in pathogen gene detection/identification}

In addition to aptamers, the next generation sequencing (NGS) system can be developed as another rapid and highly qualitative approach to identify pathogens in a high throughput manner. NGS system will be applied to characterize the unknown pathogen sequences with high accuracy. NGS is the next important sequencing tool for clinical use, as it offers unprecedented speed with improved accuracy and it is more affordable compared to Sanger sequencing (Metzker, 2005). With the reducing cost of sequencing, the NGS market is rapidly growing with a number of applications in pathogen detection. Van Dijk et al. (2014) estimated the NGS market to expand in the coming years.

NGS has many functions, such as the sequencing of an entire genome, deep sequencing for a specific target region within the genome, or even multiplex sequencing (with DNA barcode tags) which allows many samples to be sequenced simultaneously. NGS can be used to perform whole genome sequencing. A study of Influenza A and B genome has demonstrated that NGS confers $95.76 \%$ perfect matching to the reference sequence, and an accuracy of $93.7 \%$ coverage of the reference genome was attained by using consensus data (Rutvisuttinunt et al., 2013). The multiplex sequencing function is utilized so that samples from various patients who have been infected with various pathogens could be sequenced at the same time. The principle behind NGS is that signals emitted from fluorescently or radioactively labelled nucleotides are received, allowing the bases of the template DNA strand to be read sequentially. Importantly, the advantage of NGS is that it has the ability to process multiple sequencing reactions, millions in fact, in a parallel fashion. Many fragments of DNA are sequenced at the same time, which allows whole genome sequencing to be much easier with this technological advancement. Moreover, the emerging popularity of NGS is accompanied by a new automation in sample preparation methods, like microfluidics (Thaitrong et al., 2012; Kim et al., 2013). This will push the NGS into becoming an even more powerful detection tool for pathogens. It will not only detect the pathogen, but also identify the mutated sequences of newly evolved strains. Thus, NGS would be the next important sequencing tool used for biological \& clinical samples as it offers superspeed and accuracy.

The NGS promises to be transformative for the practice of microbiology and clinical disease management. The technology offers obvious advantages. Although the sequencing technology will change over time, whole genome microbial epidemiology (even with the evolution /mutations of the microbial genome) represents the ultimate source of information and will not be superseded in the foreseeable future. With the human genome information well-established and bioinformatics tools readily available, the microbial genome sequence information is easily differentiated from that of the human genome sequence. This allows us to seek out the presence of a pathogen's gene sequence amongst the human gene sequence contained within any tested samples. The implications of NGS in clinical and public health could potentiate the tracking of real-time outbreak and enables a better understanding of the epidemiology and risk factors for colonization of antimicrobial-resistant organisms in healthcare facilities or communities. With this knowledge, proactive measures can be undertaken to prevent the introduction and transmission of antimicrobial-resistant pathogens. The high throughput and deep sequencing of NGS will enlighten us on the group of microorganism, e.g. ecosystem and multiinfections within and between species, the evolution of new virulent traits, and the role of host genetics in host-pathogen interactions. As NGS could have a much lower cost at less than $\$ 1000$ per genome, this allows for deeper sequencing and high throughput efforts without loss in data quality (Schloss, 2008). Consequently, the major work of sequencing has shifted from the bench to the bedside (Hutchison, 2007). Although touted to be the tool of the new century, the analysis of the NGS data presents new challenges mainly due to the short read lengths. This problem needs to be overcome, and it will require significant investments in informatics (including hardware, software and technical support) to handle the massive amount of generated data. Nevertheless, the reality of applying NGS for pathogen detection and identification is not far off the horizon. 


\section{CONCLUSION}

Here, we have provided an update on the urgency and the next generations of pathogen detection technologies the development of aptamer technology and NGS. In antigen protein diagnosis, aptamer is more cost-effective compared with the classical antibody. Aptamer is more easily modified chemically, and produced at larger industrial scale for the detection of pathogens via their proteins or genes. NGS is more robust for sequencing small amounts of microbial genes. Larger sequence outputs, such as the entire genomes or larger targeted regions of interest, will prove to be effective to sequence through NGS, while the traditional Sanger sequencing or PCR could not run such a large number of reads at one go. Therefore, the development of the new technologies, like aptamer and NGS, specific to monitoring of infectious microbes from suspected patient samples would not only enhance the clinical treatment, but improve the survivability of the patient. It will also dramatically lower the global economic burden of the infectious disease. The new detection technologies would lower health care costs and shun the excessive hospital stay and potential over-treatment of patients. The use of aptamer, NGS and other techniques can be applied extensively for diagnostics, prognostics and therapeutics.

\section{Acknowledgements}

This work was supported by (i) TIEFA R178 Grant from Singapore Polytechnic for the promotion of the Research and Development and (ii) Ministry of Education Grant (Tier 1; R-154-000-584-112).

\section{Conflict of interest}

No conflict of interest exist.

\section{REFERENCES}

Abbaspour A, Norouz-Sarvestani F, Noori A, Soltani N. Aptamerconjugated silver nanoparticles for electrochemical dualaptamer-based sandwich detection of Staphylococcus aureus. Biosens Bioelectron. 2015. 68: 149-155.
Acquah C, Danquah MK, Yon JL, Sidhu A, Ongkudon CM. A review on immobilised aptamers for high throughput biomolecular detection and screening. Anal Chim Acta. 2015. 888: 10-18.

Akira S, Takeda K, Kaisho T. Toll-like receptors: critical proteins linking innate and acquired immunity. Nat Immunol. 2001 2: $675-680$

Bruno JG. Predicting the uncertain future of aptamer-based diagnostics and therapeutics. Molecules. 2015. 20: 6866-6887.

Cho S, Kim JE, Lee BR, Kim JH, Kim BG. Bis-aptazyme sensors for hepatitis $\mathrm{C}$ virus replicase and helicase without blank signal. Nucleic Acids Res. 2005. 33: el77.

de La Vega MA, Stein D, Kobinger GP. Ebolavirus evolution: past and present. PLoS Pathog. 2015. 11: e1005221.

Ding JL, Gan ST, Ho B. Single-stranded DNA oligoaptamers: molecular recognition and LPS antagonism are length- and secondary structure-dependent. J Innate Immun. 2009. 1: 46 -58 .

Fischer C, Hunniger T, Jarck JH, Frohnmeyer E, Kallinich C, Haase I, Hahn U, Fischer M. Food sensing: aptamer-based trapping of Bacillus cereus spores with specific detection via real time PCR in milk. J Agric Food Chem. 2015. 63: 8050 -8057 .

Fu P, Sun Z, Yu Z, Zhang Y, Shen J, Zhang H, Xu W, Jiang F, Chen $\mathrm{H}, \mathrm{Wu} \mathrm{W}$. Enzyme linked aptamer assay: based on a competition format for sensitive detection of antibodies to Mycoplasma bovis in serum. Anal Chem. 2014. 86: 1701-1709.

Hutchison CA, 3rd. DNA sequencing: bench to bedside and beyond. Nucleic Acids Res. 2007. 35: 6227-6237.

Jiang N, Tan NS, Ho B, Ding JL. Respiratory protein-generated reactive oxygen species as an antimicrobial strategy. Nat Immunol. 2007. 8: 1114-1122.

Kim H, Jebrail MJ, Sinha A, Bent ZW, Solberg OD, Williams KP, Langevin SA, Renzi RF, Van De Vreugde JL, Meagher RJ, Schoeniger JS, Lane TW, Branda SS, Bartsch MS, Patel KD. A microfluidic DNA library preparation platform for nextgeneration sequencing. PLoS One. 2013. 8: e68988.

Kotula JW, Sun J, Li M, Pratico ED, Fereshteh MP, Ahrens DP, Sullenger BA, Kovacs JJ. Targeted disruption of beta-arrestin 2-mediated signaling pathways by aptamer chimeras leads to inhibition of leukemic cell growth. PLoS One. 2014. 9: e93441.

Lai HC, Wang CH, Liou TM, Lee GB. Influenza A virus-specific aptamers screened by using an integrated microfluidic system. Lab Chip. 2014. 14: 2002-2013.

Lee $\mathrm{CH}$, Kim JH, Lee SW. Prospects for nucleic acid-based 
therapeutics against hepatitis C virus. World J Gastroenterol. 2013. 19: 8949-8962.

Lee KA, Ahn JY, Lee SH, Singh Sekhon S, Kim DG, Min J, Kim YH. Aptamer-based sandwich assay and its clinical outlooks for detecting lipocalin-2 in hepatocellular carcinoma (HCC). Sci Rep. 2015. 5: 10897.

Lee YJ, Han SR, Maeng JS, Cho YJ, Lee SW. In vitro selection of Escherichia coli O157:H7-specific RNA aptamer. Biochem Biophys Res Commun. 2012. 417: 414-420.

Li P, Wohland T, Ho B, Ding JL. Perturbation of lipopolysaccharide (LPS) micelles by Sushi 3 (S3) antimicrobial peptide. The importance of an intermolecular disulfide bond in S3 dimer for binding, disruption, and neutralization of LPS. J Biol Chem. 2004. 279: 50150-50156.

Li P, Wong JJ, Sum C, Sin WX, Ng KQ, Koh MB, Chin KC. IRF8 and IRF3 cooperatively regulate rapid interferon-beta induction in human blood monocytes. Blood. 2011. 117: 2847 -2854 .

Lin M, Li W, Wang Y, Yang X, Wang K, Wang Q, Wang P, Chang Y, Tan Y. Discrimination of hemoglobins with subtle differences using an aptamer based sensing array. Chem Commun (Camb). 2015. 51: 8304-8306.

Liu Q, Zhu Y, Yong WK, Sze NS, Tan NS, Ding JL. Synchronization of IRF1, JunB, and C/EBPbeta activities during TLR3-TLR7 cross-talk orchestrates timely cytokine synergy in the proinflammatory response. J Immunol. 2015. 195: 801 -805 .

Metzker ML. Emerging technologies in DNA sequencing. Genome Res. 2005. 15: 1767-1776.

Mosing RK, Bowser MT. Microfluidic selection and applications of aptamers. J Sep Sci. 2007. 30: 1420-1426.

Nimjee SM, Rusconi CP, Sullenger BA. Aptamers: an emerging class of therapeutics. Annu Rev Med. 2005. 56: 555-583.

Oxford JS, Bossuyt S, Lambkin R. A new infectious disease challenge: Urbani severe acute respiratory syndrome (SARS) associated coronavirus. Immunology. 2003. 109: 326-328.
Rutvisuttinunt W, Chinnawirotpisan P, Simasathien S, Shrestha SK, Yoon IK, Klungthong C, Fernandez S. Simultaneous and complete genome sequencing of influenza A and B with high coverage by Illumina MiSeq Platform. J Virol Methods. 2013. 193: 394-404.

Schloss JA. How to get genomes at one ten-thousandth the cost. Nat Biotechnol. 2008. 26: 1113-1115.

Schutze T, Wilhelm B, Greiner N, Braun H, Peter F, Morl M, Erdmann VA, Lehrach H, Konthur Z, Menger M, Arndt PF, Glokler J. Probing the SELEX process with next-generation sequencing. PLoS One. 2011. 6: e29604.

Shangguan J, Li Y, He D, He X, Wang K, Zou Z, Shi H. A combination of positive dielectrophoresis driven on-line enrichment and aptamer-fluorescent silica nanoparticle label for rapid and sensitive detection of Staphylococcus aureus. Analyst. 2015. 140: 4489-4497.

Song KM, Lee S, Ban C. Aptamers and their biological applications. Sensors (Basel). 2012. 12: 612-631.

Thaitrong N, Kim H, Renzi RF, Bartsch MS, Meagher RJ, Patel $\mathrm{KD}$. Quality control of next-generation sequencing library through an integrative digital microfluidic platform. Electrophoresis. 2012. 33: 3506-3513.

Toscano-Garibay JD, Benitez-Hess ML, Alvarez-Salas LM. Isolation and characterization of an RNA aptamer for the HPV-16 E7 oncoprotein. Arch Med Res. 2011. 42: 88-96.

Wortmann GW. Middle East respiratory syndrome: SARS redux? Cleve Clin J Med. 2015. 82: 584-588.

Yoo SM, Kim DK, Lee SY. Aptamer-functionalized localized surface plasmon resonance sensor for the multiplexed detection of different bacterial species. Talanta. 2015. 132: 112-117.

Yuce M, Ullah N, Budak H. Trends in aptamer selection methods and applications. Analyst. 2015. 140: 5379-5399.

Zhou Q, Rahimian A, Son K, Shin DS, Patel T, Revzin A. Development of an aptasensor for electrochemical detection of exosomes. Methods. 2015. 